HNO 2021 $69: 623-632$

https://doi.org/10.1007/s00106-021-01060-x

Angenommen: 15. April 2021

Online publiziert: 14. Mai 2021

(c) Springer Medizin Verlag GmbH, ein Teil von Springer Nature 2021

\author{
P. Dörig' $\cdot$ N. Gunder ${ }^{2} \cdot$ M. Witt ${ }^{3}$ A. Welge-Lüssen ${ }^{1} \cdot$ T. Hummel $^{2}$ \\ 1 Universitäts-HNO Klinik Basel, Basel, Schweiz \\ ${ }^{2}$ Universitäts-HNO Klinik Dresden, Dresden, Deutschland \\ ${ }^{3}$ Institut für Anatomie, Universitätsmedizin Rostock, Rostock, Deutschland
}

\title{
Zukunftsweisende Therapieansätze bei Riechstörungen: elektrische Stimulation, Stammzelltherapie und Transplantation von Riechepithel - eine Übersicht
}

In der Vergangenheit wurden vielfältige Therapieoptionen untersucht, diese variieren vom Riechtraining $[55,65]$ über Akupunktur [18] und medikamentöse Therapien [31, 56, 61] bis hin zur transkraniellen Magnetstimulation [28] oder, z. B. bei ausgeprägten qualitativen Riechstörungen, zur chirurgischen Resektion der Riechschleimhaut [38, 44]. Die Entwicklung einer bioelektrischen Nase, z. B. in Verbindung mit direkter elektrischer Stimulation des Bulbus olfactorius (BO), und die Transplantation von Riechschleimhaut oder von Stammzellen stellen Behandlungsmöglichkeiten der Zukunft dar.

\section{Grundlagen}

Riecheindrücke entstehen, indem Duftmoleküle an olfaktorischen Rezeptoren (OR) in den Zilien der olfaktorischen Rezeptorneurone (ORN) im Bereich des Riechepithels binden. Anschließend wird eine Kaskade aktiviert, die zur Depolarisation des ORN führt und folglich in einem Aktionspotenzial resultiert. Zur Geruchswahrnehmung wird das Aktionspotenzial über die Mitralzellen des BO in weitere Hirnareale fortgeleitet [9, 77]. Wesentlich beeinflusst wird die Geruchsempfindung zudem durch Erfahrung und physische Zustände wie z.B.
Hunger. Umgekehrt haben Gerüche auch Einfluss auf Erinnerungen und Emotionen [34].

Der Mensch besitzt ca. 370 Gene, welche OR kodieren $[24,74]$. In jedem ORN wird lediglich ein bestimmter Rezeptortyp kodiert $[63,72]$. Die jeweiligen ORN, die denselben OR besitzen, projizieren mit ihren Axonen auf nur wenige, topographisch definierte Glomeruli [42, 58, 71], dies bezeichnet man als Konvergenz, die im Riechsystem im Vergleich zu anderen Sinnessystemen sehr ausgeprägt ist. Somit entsteht ein konkretes Aktivierungsmuster im $\mathrm{BO}$ [68], anhand dessen das Gehirn in der Lage ist, die entsprechenden Duftqualitäten zu identifizieren. Es wird angenommen, dass diese sensorische Landkarte bei verschiedenen Individuen identisch ist [39].

Neben den ORN befindet sich im Riechepithel aber auch Basalzellen, die ihrerseits Vorläuferzellen für ORN, Stützzellen und mikrovilläre Zellen sind, wobei bei den Basalzellen zwischen horizontalen und globosen Basalzellen unterschieden wird [62]. Durch seine mitotische Aktivität und Differenzierungsfähigkeit erlangt die Riechschleimhaut eine lebenslange Regenerationsfähigkeit, was für ein menschliches Sinnesepithel einzigartig ist. Die Aufgabe der mikrovillären Zellen ist letztlich noch nicht 
geklärt, sie dienen aber auch sensorischen Funktionen. Die der Stützzellen liegt u. a. in der metabolischen Unterstützung der Funktion der ORN. Im Rahmen von COVID-19-assoziierten Riechstörungen scheinen sie ebenfalls eine zentrale Rolle $\mathrm{zu}$ spielen[12]. In der Lamina propria der Riechschleimhaut befinden sich zudem „olfactory ensheathing cells“, ein Gliazelltyp, welche die olfaktorischen Rezeptorneuronen umgeben. Sie gewährleisten elektrische Isolierung und reichen von der Peripherie, und nach Durchtritt durch die ca. 1-2 mm großen Öffnungen der Lamina cribrosa, bis ins zentrale Nervensystem (ZNS) zum BO $[16,64,78]$.

Abgesehen vom Alter stellen Infekte, chronische sinunasale Erkrankungen, Schädel-Hirn-Traumen oder neurodegenerative Krankheiten die häufigsten Ursachen von Riechstörungen dar [20]. In der Folge kann das Riechvermögen vollständig erloschen sein, was man als Anosmie bezeichnet. In Abhängigkeit von der Genese der Riechstörung können die Beschwerden vorübergehend oder konstant sein. Hinsichtlich COVID-19assoziierter Riechstörungen ist bemerkenswert, dass häufig eine signifikante Besserung bereits innerhalb von 2 Wochen auftritt [7]. Zudem wurde schon rasch bemerkt, dass die Beeinträchtigung der Riechfunktion ein Frühsymptom darstellt und dieses typischerweise um den vierten Tag der Erkrankung auftritt. Der plötzliche Beginn der Riechstörung ist für eine postinfektiöse Riechstörung eher typisch [50]. Da SARS-CoV-2-Patienten selten über zusätzliche rhinitische Beschwerden klagen, legen pathophysiologische Überlegungen nahe, dass es sich nicht primär um eine konduktive Problematik handelt, sondern eher von einer Schädigung des Riechepithels oder von einer neuronalen Affektion ausgegangen werden muss [32]. Diese Annahme unterstützt die zwischenzeitlich gewonnene Erkenntnis, dass SARSCoV-2 über den Oberflächenrezeptor ACE2 in die Zelle gelangt, welcher v.a. von den Stützzellen exprimiert wird. Dies wäre auch eine mögliche Erklärung für die rasche Verbesserung der Riechfunktion, da die ORN selbst nur indirekt betroffen sind [12]. Diskutiert wird zu- dem ein möglicher Eintritt des Virus über Neuropilin 1 ins Riechepithel und in das Gehirn, sodass COVID-19-assoziierte Riechstörungen auch durch eine Beeinträchtigung der verarbeitenden Zentren im Gehirn zustande kommen könnten [32]. Am Beispiel von SARSCoV-2 wird ersichtlich, dass die Ursache der Riechstörung auch Einfluss auf die Verbesserung der olfaktorischen Funktion im Verlauf hat [76]. Trotz vielseitiger Bemühungen, eine suffiziente Therapie für Riechstörungen $\mathrm{zu}$ entwickeln, sind die Erfolge auf diesem Gebiet begrenzt.

Verliert ein Mensch eine ganze Sinnesqualität, stellt dies eine erhebliche Beeinträchtigung für das alltägliche Leben des Betroffenen dar. In Bezug auf das Hören lässt sich die angeborene sowie erworbene hochgradige, an Taubheit grenzende Schwerhörigkeit mithilfe eines Cochleaimplantats oft relativ gut behandeln $[45,53]$. Eine Analogie für erblindete Personen stellt das Retinaimplantat zur Behandlung degenerativer retinaler Erkrankungen dar. Das erste Produkt dafür wurde 2013 in den USA zugelassen [57]. Diese Arbeiten zeigen, dass eine elektrische Stimulation von Sinnesorganen bzw. Sinnesnerven zu Empfindungen führen kann, die in der Orientierung im Alltag eine außerordentlich große Hilfe sein können.

Neben einer elektrischen Stimulation könnte ein anderer zukunftsweisender Therapieansatz in der funktionellen Wiederherstellung des Riechepithels liegen. Die Idee, defekte Organsysteme durch gesunde zu ersetzen, ist nicht neu. Hauttransplantationen wurden in Asien bereits 600 vor Christus beschrieben [2]. Die erste erfolgreiche Nierentransplantation erfolgte in Paris schon im Jahr 1953 [27]. Am Beispiel von hämatoonkologischen Patienten lässt sich zudem zeigen, wie groß der Stellenwert von Stammzelltransplantationen in unseren heutigen Therapiekonzepten ist [4]. Solche Therapieansätze bieten sich bei der Behandlung von Riechstörungen besonders an, da in der gesunden Riechschleimhaut natürlicherweise Stammzellen vorkommen und sich aus selbigen das Riechepithel regeneriert.

Die vorliegende Übersicht soll einen Einblick in das Thema der elektrischen
Stimulation des olfaktorischen Systems und die Realisierbarkeit einer bioelektrischen Nase geben. Zudem werden aktuelle wissenschaftliche Erkenntnisse hinsichtlich einer möglichen Transplantation von Riechschleimhaut sowie einer Stammzelltherapie zur Wiederherstellung der Riechfunktion dargestellt.

\section{Elektrische Stimulation am olfaktorischen System}

\section{Elektrische olfaktorische Aktivierung bei Tieren}

Bereits 1959 veröffentlichte Ottoson eine Arbeit zur elektrischen Stimulation der nasalen Schleimhaut beim Frosch. Durch diese ließen sich Potenzialänderungen von $\mathrm{N}$. olfactorius und $\mathrm{BO}$ auslösen. Es wurden verschiedene Areale der Mukosa stimuliert. Lediglich Aktivierungen im Bereich der Riechschleimhaut führten zu einer Potenzialveränderung im BO [51]. In dieser Studie konnte grundlegend dargestellt werden, dass die elektrische Stimulation des $\mathrm{N}$. olfactorius sowie des $\mathrm{BO}$ realisierbar sind.

Coelho und Constanzo führten Untersuchungen mittels einer 32-KanalElektrode zur räumlichen Kartierung des BO bei Ratten durch. Zur Reizung verwendeten sie natürliche Düfte und direkte elektrische Stimulation. Dabei registrierten sie die Reizantworten auf die jeweiligen Stimuli in verschiedenen Arealen des BO. Mithilfe verschiedener Programme ließen sich für die natürlichen Duftstoffe Muster generieren, welche die entsprechenden neuronalen Aktivitäten für die jeweiligen Duftqualitäten darstellen. So konnten die Autoren nachweisen, wie in vorhergehenden Studien auch, dass unterschiedliche Gerüche zu lokalisierten Aktivierungsmustern im BO führen. Auch für die direkte elektrische Stimulation ließ sich in Abhängigkeit von der Lokalisation der Reizung eine lokalisierte Reaktion ableiten [14].

Um die neuronale Aktivität der ORN nach Präsentation eines olfaktorischen oder evtl. auch elektrischen Reizes nachvollziehbar zu machen, implantierten Dong et al. eine selbst hergestellte 16Kanal-Mikroelektrode im BO bei Ratten. 
Fünf verschiedene Düfte (Anisol, Citral, Carvon, Isobutanol, Isoamylacetat) führten zu unterschiedlichen neuronalen Aktivitäten der ORN, und es ließen sich in Abhängigkeit von der Reizung mit den unterschiedlichen Düften unterschiedliche Aktivierungsmuster darstellen. Durch einen Dekodierungsalgorithmus konnten die Duftstoffe identifiziert werden [22]. Anhand dieser Arbeit konnte letztlich die Machbarkeit der Unterscheidung von Geruchsqualitäten durch eine bioelektrische Nase demonstriert werden. Allerdings ergaben sich Schwierigkeiten bei der Unterscheidung von Duftgemischen sowie unterschiedlicher Duftkonzentrationen.

\section{Elektrische Reizung der menschlichen Riechschleimhaut}

Erste Untersuchungen zur Stimulation der Riechschleimhaut beim Menschen wurden bereits im 19. Jahrhundert veröffentlicht. Dabei wurden durch die elektrische Reizung über eine endonasal platzierte Elektrode Geruchsempfindungen erzeugt. Zum Beispiel beschrieben die Probanden den wahrgenommenen Geruch als ein "angezündetes Streichholz“ [3].

Uziel publizierte 1973 die Ergebnisse seiner Versuche, bei denen jeweils Teilnehmenden unter Sicht zwei verschiedene Elektroden, eine Silber- sowie eine beschichtete Silber-SilberchloridNatriumchlorid(Ag-AgCl-NaCl)-Elektrode, im Bereich der olfaktorischen Schleimhaut ( Abb. 1a, b) platziert wurden. Bei der anodischen Stimulation beobachtete Uziel die Entstehung olfaktorischer Empfindungen definiert durch Schwelle, Latenzzeit, Dauer und Qualität. Die Duftqualität wurde von vier Probanden als „Mandel“ beschrieben, ein weiterer berichtete über eine „verbrannte " Geruchsempfindung. Dem entgegen ließen sich anhand der kathodischen Stimulation und durch die Ag-AgCl-NaClElektrode lediglich trigeminale Empfindungen, wie "Brennen“ und „Stechen“ hervorrufen. Jedoch wurden die Beobachtungen der anodischen Stimulation auf die Freisetzung von Chlor zurückgeführt, das die Empfindung ausgelöst haben könnte [69].

HNO 2021 · 69:623-632 https://doi.org/10.1007/s00106-021-01060-x

(c) Springer Medizin Verlag GmbH, ein Teil von Springer Nature 2021

P. Dörig · N. Gunder · M. Witt · A. Welge-Lüssen · T. Hummel

Zukunftsweisende Therapieansätze bei Riechstörungen: elektrische Stimulation, Stammzelltherapie und Transplantation von Riechepithel - eine Übersicht

\section{Zusammenfassung}

Passagere oder permanente Riechstörungen können verschiedene Ursachen haben. Ganz aktuell berichtet eine Vielzahl von Patienten im Rahmen von COVID-19-Infektionen über ein fehlendes oder vermindertes Riechvermögen. In der Vergangenheit wurden vielfältige Therapieoptionen untersucht, diese variieren vom Riechtraining über Akupunktur und medikamentöse Therapien bis hin zur transkraniellen Magnetstimulation oder, z. B. bei ausgeprägten qualitativen Riechstörungen, der chirurgischen Resektion der Riechschleimhaut. Die Entwicklung einer bioelektrischen Nase, z. B. in Verbindung mit direkter elektrischer Stimulation des Bulbus olfactorius, oder die Transplantation von Riechschleimhaut oder von Stammzellen stellen Behandlungsmöglichkeiten der Zukunft dar. Die Grundlagen für diese Entwicklungen sowie der Stand des Wissens werden in der vorliegenden Arbeit erläutert.

\section{Schlüsselwörter}

Anosmie · Nase · Geruch · Behandlung ·

Implantat · Transplantat

\section{Future therapeutic strategies for olfactory disorders: electrical stimulation, stem cell therapy, and transplantation of olfactory epithelium -an overview}

Abstract

Olfactory disorders may be temporary or permanent and can have various causes. Currently, many COVID-19 patients report a reduced or complete loss of olfactory function. A wide range of treatment options have been investigated in the past, such as olfactory training, acupuncture, medical therapy, transcranial magnetic stimulation, or surgical excision of olfactory epithelium, e.g., in severe qualitative smell disorders. The development of a bioelectric nose, e.g., in connection with direct electrical stimulation or transplantation of olfactory epithelium or stem cells, represent treatment options of the future. The basis of these developments and the state of knowledge is discussed in the following work.

Keywords

Anosmia $\cdot$ Nose $\cdot$ Smell · Treatment $\cdot$ Implant . Graft
In einer Untersuchung von Straschill et al. führte die elektrische Stimulation der olfaktorischen Mukosa zu einer Unterdrückung der Wahrnehmung von Düften. Weiterhin wurde beobachtet, wenn ein Elektrostimulus in einem bestimmten Intervall nach einem Duftstimulus appliziert wurde, dass dieser zu einer ähnlichen Geruchswahrnehmung führte wie der zuvor dargebotene Duft. Elektrische Stimulationen ohne vorausgegangenen Duftreiz führten außerdem bei drei Patienten zu einer „kakosmischen" Wahrnehmung - dies waren Patienten mit Temporallappenepilepsie und olfaktorischen Auren [66].

Ishimaru et al. registrierten erstmals 1997 EEG-abhängige evozierte Poten- ziale nach elektrischer Stimulation der menschlichen Riechschleimhaut. Bei fünf Probanden wurde die olfaktorische Schleimhaut unilateral über eine bipolare Ag-Elektrode elektrisch gereizt. Gleichzeitig wurden die Hirnströme mit jeweils zwei Elektroden (eine frontal und eine lateral) registriert. Zwar ließen sich nach der elektrischen Stimulation Potenziale registrieren, jedoch wurde keine Geruchsempfindung angegeben. Reizungen außerhalb der Riechspalte lösten eine Schmerzempfindung aus, am ehesten aufgrund einer Affektion des N. trigeminus [33].

Weiss et al. führten 2016 verschiedene Versuchsanordnungen bei 60 Freiwilligen durch, wobei elektrische Reize über 

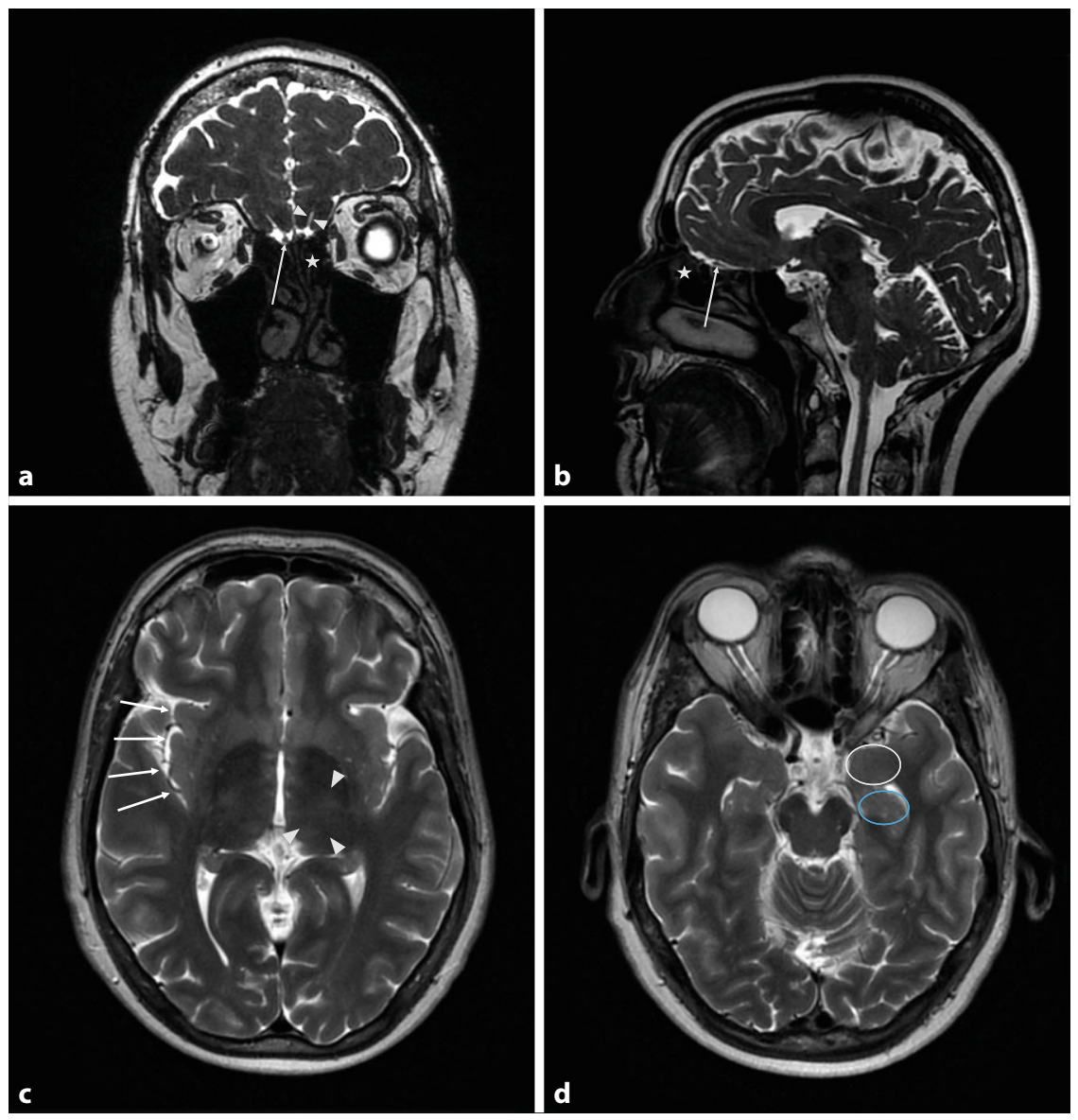

Abb. 1 a a Magnetresonanztomographie (MRT) des Schädels; koronares T2-gewichtetes Bild. Bulbus olfactorius (Pfeil), Sulcus olfactorius (gefülltes Dreieck), Riechschleimhaut (Stern). b MRT des Schädels; sagittales T2-gewichtetes Bild. Bulbus olfactorius (Pfeil), Riechschleimhaut (Stern). c MRTdes Schädels; axiales T2-gewichtetes Bild. Insula (Pfeile), Thalamus (gefüllte Dreiecke). d MRT des Schädels; axiales T2gewichtetes Bild. Amygdala (weißer Kreis), Hippocampus (blauer Kreis)

eine intranasale Ag-Elektrode appliziert wurden. In der ersten Versuchsanordnung konnte die Hypothese, dass eine Stimulation im Bereich der Riechschleimhaut zu einer Geruchswahrnehmung führt, anhand von Stimulation verschiedener Areale der Nase (mittlere und obere Muschel, Septumhinterkante und Riechspalte) nicht bestätigt werden. Zu keinem Zeitpunkt berichteten die Probanden über einen Geruchseindruck, allerdings führte die gleichzeitige elektrische Stimulation im Bereich der Riechschleimhaut zu einer Modulation eines Riecheindrucks. Angenehme Gerüche (Rose und Schokolade) wurden durch die gleichzeitige elektrische Stimulation statistisch signifikant weniger angenehm empfunden. Zusätzlich unterzogen sich 20 Teilnehmende einer funktionellen MRT, um den Effekt der elektrischen Stimulation der Riechschleimhaut bildmorphologisch darzustellen. Dabei konnte eine neuronale Reaktion speziell im primären Riechkortex dargestellt werden, was zwar auf die korrekte Lage der Elektrode in der Riechspalte deutete, dennoch konnte keine olfaktorische Wahrnehmung durch die elektrische Stimulation der Riechschleimhaut erzeugt werden [75].

In der Summe zeigen diese Untersuchungen, dass die direkte elektrische Stimulation der Riechschleimhaut zu olfaktorischen Wahrnehmungen führen kann. Darüber hinaus führt die elektrische Stimulation im Bereich der Riechspalte zu einer Modulation von dargebotenen Düften. Die Wahrnehmung dieser kann unterdrückt, aber auch nachträglich wieder hervorgerufen werden.
Elektrische Reizung des BO beim Menschen

Die direkte elektrische, intraoperative Reizung des BO (• Abb. 1a, b) am wachen Patienten führte bei Untersuchungen von Penfield und Jasper zu einer Wahrnehmung von eher unangenehmen Gerüchen wie verbranntem Gummi oder Gülle [52]. Eine Stimulation des BO kann aber auch erreicht werden, indem die Elektroden im Bereich der Lamina cribrosa platziert werden. Nach Positionierung einer Testelektrode im Bereich der Lamina lateralis der Lamina cribrosa führte dort die elektrische Stimulation bei drei Probanden zu einer subjektiven Geruchswahrnehmung. Diese wurden als zwiebelartig oder antiseptisch und sauer oder fruchtig und schlecht beschrieben. Damit stellt die transethmoidale Stimulation des BO einen neuartigen Ansatz bei der Entwicklung der bioelektrischen Nase dar [30].

\section{Elektrische Reizung des Kortex und anderer Hirnareale des Menschen}

Orbitofrontaler Kortex: Kumar et al. veröffentlichten eine Studie, in der sie 16 Kindern mit einer fokalen Epilepsie subdurale Elektroden rechts oder/und links frontal, temporal, parietal und okzipital implantierten. Elektrische Stimulationen im ventralen Bereich des medialen Frontallappens führten bei elf Kindern zu einer Geruchswahrnehmung. Diese Geruchsempfindung wurde meist als unangenehm, Rauch oder Müll beschrieben. Zwei Kinder berichteten von angenehmen Gerüchen wie Erdbeeren oder gutem Essen. Elektrische Reizungen anderer Hirnareale führten nicht zu Riecheindrücken. Olfaktorische „Halluzinationen“ ließen sich lediglich durch Stimulationen in der Nähe des BO oder des Tractus olfactorius erzeugen [35].

Anders als zuvor beschrieben, gelang es Bérard et al., angenehme Riecheindrücke durch die Elektrostimulation des medialen orbitofrontalen Kortex $\mathrm{zu}$ erzeugen. Im Rahmen dieser Studie wurden elektrische Impulse über verschieden lokalisierte Tiefenelektro- 
Tab. 1 Übersicht zur elektrischen Stimulation am olfaktorischen System

\begin{tabular}{lll}
\hline $\begin{array}{l}\text { Elektrische olfaktorische Aktivierung bei Tieren } \\
\text { Ottoson (1959) [51] }\end{array}$ & Frösche & \multicolumn{1}{l}{ eStim Nasenschleimhaut } \\
$\begin{array}{l}\text { Coelho und Costan- } \\
\text { zo (2016) [14] }\end{array}$ & $\begin{array}{l}\text { Duftstimuli Aufzeichnung neuro- } \\
\text { naler Aktivität im BO }(n=8) \\
\text { eStim des BO }(n=4)\end{array}$ \\
$\begin{array}{l}\text { Dong et al. (2013) } \\
\text { [22] }\end{array}$ & $\begin{array}{l}\text { eStim durch 16-Kanal-Mikroelek- } \\
\text { trode am BO } \\
\text { Präsentation fünf verschiedener } \\
\text { Düfte }\end{array}$
\end{tabular}

Elektrische Reizung der menschlichen Riechschleimhaut

\begin{tabular}{|c|c|c|}
\hline Aronsohn (1886) [3] & 4 Gesunde & $\begin{array}{l}\text { Beidseits endonasal platzierte } \\
\text { Elektrode }\end{array}$ \\
\hline Uziel (1973) [69] & $\begin{array}{l}21 \text { (Gesunde und } \\
\text { HNO-Patienten) }\end{array}$ & $\begin{array}{l}\text { eStim mit diversen Elektroden ( } \mathrm{Ag} \\
\text { und } \mathrm{Ag}-\mathrm{AgCl}-\mathrm{NaCl})\end{array}$ \\
\hline
\end{tabular}

Straschill et al. 10 Gesunde, 5 Epi- eStim und Präsentation von Duft(1983) [66] leptiker stimuli

Ishimaru et al. 5 Gesunde eStim über Ag-Elektrode

(1997) [33]

Weiss et al. (2016) 60 Gesunde

[75]

schnitte
fMRT $(n=20)$

Elektrische Reizung des BO beim Menschen

\begin{tabular}{lll}
$\begin{array}{l}\text { Penfield und Jasper } \\
\text { (1954) [52] }\end{array}$ & 5 Epileptiker & $\begin{array}{l}\text { Intraoperative eStim des BO am } \\
\text { wachen Patienten }\end{array}$ \\
\hline $\begin{array}{l}\text { Holbrook et al. } \\
\text { (2019) [30] }\end{array}$ & 5 Patienten mit CRS & \begin{tabular}{l} 
Transethmoidale eStim des BO \\
\hline
\end{tabular}
\end{tabular}
(2019) [30]

Elektrische Reizung des Kortex und anderer Hirnareale des Menschen

Kumar et al. (2012) 16 Kinder mit Epilep- eStim via subdurale Elektroden

[35] sie

Bérard et al. (2020) 8 Epileptiker

[5]

Mazzola et al. (2017) 221 Epileptiker [40]
Andy (1967) [1] 1 Epileptiker eStim Amygdala und Hippocam- pus (rechtsseitig)

Nashold und Wilson 5 mit neurologischer eStim via Tiefenelektroden am (1969) [48] Erkrankung wachen Patienten eStim via Tiefenelektroden eStim der Insula via Tiefenelektroden

Stimulation im Bereich der Riechschleimhaut $\rightarrow$ Potenzialänderungen im BO

Lokalisierte Aktivierungsmuster im BO durch verschiedene Duftstimuli

eStim an verschiedenen Lokalisationen des $\mathrm{BO} \rightarrow$ lokalisierte Aktvierung des $\mathrm{BO}$ mittels evozierter Potenziale

Ableitung geruchsabhängiger Aktvierungsmuster der ORN Duftidentifizierung durch Dekodierungsalgorithmus der Aktivierungsmuster $(n=4)$

Geruchswahrnehmung „Streichholz” $(n=2)$

Anodische Stimulation $\rightarrow$ Geruchswahrnehmung „Mandel“ ( $n=5)$, "Verbrannt" $(n=3)$, Vanille $(n=1)$ und "eitrig" $(n=1)$ Ag-AgCl-NaCl: keine olfaktorische Sensation, jedoch Schmerzempfindung

Unterdrückung der Geruchswahrnehmung

Kakosmische Riecheindrücke ohne Duftstimulus $(n=3)$

Keine Erzeugung von olfaktorischen Empfindungen

Modulation dargebotener Duftstimuli lediglich bei eStim des Riechepithels

Keine Generation olfaktorischer Empfindungen durch eStim

Unangenehme Geruchsempfindung

Geruchswahrnehmungen ( $n=3)$ : zwiebelartig, antiseptisch, sauer, fruchtig, schlecht

Stimulation nahe BO oder Tractus olfactorius $\rightarrow$ Riecheindrücke $(n=13)$

Stimulation am medialen orbitofrontalen Kortex $\rightarrow$ Geruchswahrnehmung $(n=5)$

Geruchsempfindungen durch Reize an mediodorsaler Insula

Elektrostimulation der Amygdala $\rightarrow$ olfaktorische Empfindung (faulig), Elektrostimulation des Hippocampus ohne Geruchsempfindung Elektrostimulation des Thalamus $\rightarrow$ diverse Riecheindrücke (Gummi, rauchig/verbrannt, Chloroform, Nelke)

Ag Silber, Ag-AgCl-NaCl Silber-Silberchlorid-Natriumchlorid, BO Bulbus olfactorius, CRS chronische Rhinosinusitis, eStim elektrische Stimulation, fMRT funktionelle Magnetresonanztomographie, $n$ Anzahl

den bei acht Patienten mit einer Temporallappenepilepsie appliziert. Nach Stimulationen im Bereich des Sulcus olfactorius, des Sulcus orbitalis medialis oder des Gyrus orbitofrontalis medialis berichteten fünf der acht Probanden über angenehme Riecheindrücke. Beispielsweise wurden diese als Zitrone, Kaffee, Parfüm, Eukalyptus und Obst oder Gemüse beschrieben. Eine Änderung der Stimulationsamplitude führte zu einer
Änderung der Geruchswahrnehmung bei drei der fünf Patienten [5].

Insulärer Kortex: Anhand retrospektiver Untersuchungen von elektrischen Stimulationen der Insula (• Abb. 1c) konnte diese hinsichtlich der ausgelösten Riech- und Schmeckempfindungen kartographiert werden. Es wurden 651 Untersuchungen ausgewertet, bei denen die Insula von 221 Patienten mit einer medikamentenrefraktären Epilep- sie über eine stereotaktisch implantierte Tiefenelektrode stimuliert wurde. Sechsmal wurde eine Geruchsempfindung angegeben, welche durch elektrische Stimulationen im Bereich der mittleren dorsalen Insula ausgelöst wurde. Weiterhin zeigten die Ergebnisse, dass es eine räumliche Überlappung zwischen gustatorischer, olfaktorischer und oraler somatosensorischer Repräsentation in diesem Bereich gibt [40]. 
Amygdala und Hippocampus: Andy veröffentlichte 1967 den Fallbericht eines Patienten, der nach elektrischer Stimulation der rechten Amygdala (• Abb. 1d) von einer üblen Geruchswahrnehmung berichtete. Durch Stimulation des Hippocampus ( $\bullet$ Abb. 1d) wurde allerdings keine Geruchsempfindung erzeugt [1].

Thalamus: Auch die Elektrostimulation des Thalamus (• Abb. 1c) kann olfaktorische Halluzinationen hervorrufen. Im Rahmen der Untersuchungen von Nashold und Wilson ließen sich bei drei von fünf Probanden unangenehme Geruchswahrnehmungen erzeugen (verbrannt, verbranntes Gummi oder Chloroform). Ein Teilnehmender nahm angenehme Gerüche wie Heu und Nelke wahr. Weiterhin berichtete ein Proband über eine vage Geruchsempfindung, die ihn an "South Dakota“ erinnerte [48].

Die Untersuchungen legen also nahe, dass elektrische Stimulation u.a. in Abhängigkeit von Stimulationsparametern und dem Reizort zu verschiedenen Geruchsempfindungen führen kann. Zusammenfassend sind die verschiedenen Applikationsorte und erreichten Ergebnisse in $\bullet$ Tab. 1 dargestellt.

\section{Neurogenese der Riechschleim- haut}

Andere therapeutische Bestrebungen setzen an der direkten Funktionswiederherstellung des geschädigten Riechepithels an. Eine wichtige Rolle kommt hierbei den ORN zu, welche sowohl für die Rezeption von Gerüchen als auch für die Signalweiterleitung verantwortlich sind. Folglich sind Kenntnisse über die Regeneration und die Neurogenese dieser Zellen für die Entwicklung und das Verständnis solcher Behandlungsansätze zentral.

Bereits im 19. Jahrhundert gab es Konzepte zur Charakterisierung von Zellen bei Wirbeltieren in Abhängigkeit von deren Regenerationsfähigkeit. In Bezug auf Nervenzellen wurde damals davon ausgegangen, dass sie ein stabiles Zellverhalten zeigen und entsprechend nicht ersetzt werden können, selbst dann nicht, wenn sie zerstört werden. Aufgrund von fehlenden Untersuchungen am Riechepithel hatte sich diese Vorstellung relativ lange gehalten. In den späten 1970er-Jahren hat sich das Verständnis aber grundlegend geändert. Mithilfe des radioaktiv markierten ${ }^{3} \mathrm{H}$-Thymidins, einem Thymidinanalogon, konnte gezeigt werden, dass sich teilungsfähige Vorläuferzellen in ORN differenzieren und so eine lebenslange neurogenetische Matrix bei Wirbeltieren darstellen. Nach Nervendurchtrennung von ORN konnte zudem eine Nervenzellregeneration beobachtet werden. Im Rahmen der Regeneration kam es sogar zu einer Axonaussprossung in Richtung Vorderhirn auch dann, wenn den untersuchten Mäusen der BO entfernt worden war [25].

Anhand von Nasenschleimhautbiopsien, welche bei Autopsien sowie Nasenoperationen entnommen wurden, untersuchten Murrell et al. die Neurogenese der olfaktorischen Schleimhaut beim Menschen. Hierfür legten sie serumfreie Zellkulturen an und stimulierten diese mit Fibroblastenwachstumsfaktor 2 (FGF-2). Innerhalb der ersten 15-20 Tage nach Stimulation kam es zu einer deutlichen Zunahme an bipolaren Zellen, die immunhistochemisch positiv für das olfaktorische Markerprotein (OMP) waren, welches typischerweise bei reifen ORN zu finden ist. Mittels radioaktiv markierten Thymidins wurde schließlich in vitro die Differenzierung in ORN nachgewiesen. Das Alter des ältesten Probanden, bei dem eine Biopsie entnommen wurde, betrug 72 Jahre, sodass im Rahmen der Arbeit eine Neurogenese in der menschlichen Riechschleimhaut bis ins höhere Alter nachgewiesen werden konnte [47]. Eine kürzlich veröffentlichte Arbeit bestätigt, dass beim Menschen bis ins Erwachsenenalter eine Neurogenese in der Riechschleimhaut stattfindet. Die Autoren konnten belegen, dass bei den untersuchten Probanden (Alter 41-52 Jahre) verschiedene neurogene Zellstadien vorhanden sind. Im Unterschied zu Nagern war der Anteil der unreifen Rezeptorneuronen mit 55\% aber eher hoch, wohingegen bei Nagern dieser Teil nur 5-15\% ausmacht [23]. Dass die neuroregenerative Kapazität aber nicht lebenslang uneingeschränkt vorhanden, sondern eine gewisse Ermüdung anzunehmen ist, wurde an einem transgenen Mausmodell dargestellt. In den genetisch veränderten Tieren stellte sich ein vorzeitiger Zelluntergang von ORN ein, was wiederum zu einem erhöhten Zellumsatz führte. Bereits nach zwei Monaten glich die Riechschleimhaut derjenigen von älteren Menschen mit Bereichen ohne Neuronen oder globose Basalzellen, welche eine Grundvoraussetzung für die neuronale Regeneration darstellen. In diesen Arealen waren zudem Gewebeveränderungen im Sinne einer respiratorischen Metaplasie zu beobachten. Als Konsequenz dieser Veränderung, kommt es zu einer verminderten neuronalen Stimulation des BO, was auch in einer Volumenminderung (Verkleinerung) der Glomeruli zu erkennen war [13]. Dass eine olfaktorische Deprivatisierung mit einer Volumenminderung des BO einhergeht, konnte vor vielen Jahren auch schon von Meisami et al. gezeigt werden [41]. Die Neurogenese in der Riechschleimhaut ist nicht nur vom Alter, sondern auch von der olfaktorischen Exposition eines Individuums abhängig. In einer Untersuchung an Mäusen wurde festgestellt, dass olfaktorische Stimulierung eine Hochregulierung von gewissen Subtypen von ORN zur Folge hat, was einen adaptiven und plastischen Charakter der Riechschleimhaut nahelegt [70]. Zusätzlich scheint innerhalb der olfaktorischen Mukosa eine räumliche Determination hinsichtlich der Ausreifung der ORN zu bestehen. Coleman und seine Arbeitsgruppe entnahmen bei Mäusen Vorläuferzellen im dorsalen Bereich der Riechschleimhaut und setzten diese weiter ventral wieder in die olfaktorische Schleimhaut ein. Sie zeigten, dass nach erfolgtem Transfer ein Großteil der Neuronen typischerweise ventral aufzufindende Oberflächenglykoproteine exprimierten, dafür aber die Expression bestimmter Enzyme verloren [15]. Für die Regeneration und Plastizität der Riechschleimhaut sind also verschiedene Faktoren verantwortlich.

Die Riechschleimhaut hat ein enormes regeneratives Potenzial. Selbiges wird nicht nur vom Alter, sondern von verschiedenen anderen Faktoren beeinflusst. Diese Plastizität der Schleimhaut könnte auch in einem therapeutischen 


\begin{tabular}{|c|c|c|c|}
\hline Quelle (Jahr) & $\begin{array}{l}\text { Studien- } \\
\text { population }\end{array}$ & Methodik & Ergebnis \\
\hline \multicolumn{4}{|c|}{ Transplantation von Stammzellen } \\
\hline $\begin{array}{l}\text { Tsujigiwa et al. } \\
\text { (2005) [67] }\end{array}$ & Mäuse & $\begin{array}{l}\text { aTrans von GFP-positiven-Knochenmarkzellen } \\
\text { durch venöse Injektion }\end{array}$ & $\begin{array}{l}\text { Markierte Knochenmarkzellen migrieren in die Riechschleimhaut des } \\
\text { Empfängers und differenzieren sich teilweise in ORN }\end{array}$ \\
\hline $\begin{array}{l}\text { Ochi et al. } \\
\text { (2010) [49] }\end{array}$ & Mäuse & $\begin{array}{l}\text { aTrans von BrdU-markierten Knochenmark- } \\
\text { stammzellen durch venöse oder transnasale } \\
\text { Injektion }\end{array}$ & $\begin{array}{l}\text { Vergleichbare Effizienz der Zelltransplantationsanwachsrate im } \\
\text { Riechepithel unabhängig der Applikationsform. } \\
\text { Keine Ausreifung von Vorläuferzellen in ORN }\end{array}$ \\
\hline $\begin{array}{l}\text { Diaz et al. } \\
\text { (2012) [21] }\end{array}$ & Mäuse & $\begin{array}{l}\text { aTrans von Knochenmarkstammzellen durch } \\
\text { venöse Injektion in transgene Mäuse mit er- } \\
\text { höhtem Mitralzellverlust im BO }\end{array}$ & $\begin{array}{l}\text { Ausbildung von zahlreichen Mikrogliazellen im BO mit neuroprotek- } \\
\text { tivem Effekt } \rightarrow \text { reduzierter Verlust von Mitralzellen } \rightarrow \text { Verbesserung } \\
\text { von Geruchserkennung sowie Geruchsdiskrimination }\end{array}$ \\
\hline $\begin{array}{l}\text { Kurtenbach } \\
\text { et al. (2019) } \\
{[36]}\end{array}$ & Mäuse & $\begin{array}{l}\text { aTrans von Stammzellen der Riechschleimhaut } \\
\text { in transgene hyposmische Empfänger mittels } \\
\text { nasaler Injektion }\end{array}$ & $\begin{array}{l}\text { Ausbildung von zilientragenden ORN inkl. Axonaussprossung bis in } \\
\text { den } \mathrm{BO} \rightarrow \text { verbesserte Riechfunktion }\end{array}$ \\
\hline \multicolumn{4}{|c|}{ Transplantation von Riechschleimhaut } \\
\hline $\begin{array}{l}\text { Morrison et al. } \\
\text { (1983) [43] }\end{array}$ & Ratten & $\begin{array}{l}\text { aTrans von neonatalem } \mathrm{OE} \text { in den parietalen } \\
\text { Kortex und den vierten Ventrikel }\end{array}$ & $\begin{array}{l}\text { Fortbestehen der Neurogenese im Transplantat an ektoper Lage. } \\
\text { Ausbildung von Axonen, jedoch nicht von olfaktorischen Glomeruli }\end{array}$ \\
\hline $\begin{array}{l}\text { Holbrook et al. } \\
\text { (2001) [29] }\end{array}$ & Mäuse & $\begin{array}{l}\text { aTrans von postnatalem } \mathrm{OE} \text { in den parietalen } \\
\text { Kortex }\end{array}$ & $\begin{array}{l}\text { Transplantatüberleben in } 85 \% \text { mit Erhalt von regenerativer Epithel- } \\
\text { funktion }\end{array}$ \\
\hline $\begin{array}{l}\text { Yagi et al. } \\
\text { (2009) [79] }\end{array}$ & Mäuse & $\begin{array}{l}\text { aTrans von postnatalem OE in den zerebellären } \\
\text { Kortex }(n=12) \text { sowie den } \mathrm{BO}(n=6)\end{array}$ & $\begin{array}{l}\text { Transplantatüberlebensrate an beiden Orten bei } 83 \% \text {. Transplantat } \\
\text { weist Epithelcharakteristika von normalem OE auf }\end{array}$ \\
\hline
\end{tabular}

Kontext genutzt werden. Im Folgenden wird der therapeutische Einsatz von Stammzellen dargestellt und zudem auf die Möglichkeit einer Transplantation von Riechschleimhaut eingegangen.

\section{Transplantation von Stammzellen}

Das Knochenmark enthält eine heterogene Zellpopulation mit multipotenten Stammzellen, Vorläuferzellen und ausdifferenzierten Zellen. Die hohe Plastizität der Knochenmarkzellen erlaubt diesen, verschiedene Gewebe zu besiedeln und sich in diverse Zelllinien zu differenzieren. Diese Potenz macht Knochenmarkzellen zu einem guten Kandidaten für Zelltherapien [21].

Tsujigiwa hat bereits 2005 in einer Arbeit gezeigt, dass transplantierte Knochenmarkzellen in die Riechschleimhaut des Empfängers migrieren, dort anwachsen und sich auch in olfaktorische Neuronen differenzieren können. Hierfür hat er Knochenmarkzellen von transgenen Mäusen, welche grün fluoreszierendes Protein (GFP) exprimierten, in vorgängig bestrahlte Mäuse transplantiert. Bereits zwei Wochen nach Transplantation konnten GFP-positive Zellen in der olfaktorischen Mukosa nachgewiesen werden. Nach weiteren 3,5 Monaten waren die Zellen nicht nur in der basalen Schicht der Riechschleimhaut zu finden, sondern auch in der mittleren Schleimhautschicht, wo sich typischerweise reife olfaktorische ORN befinden. Durch immunhistochemische Analysen für OMP konnte außerdem der Nachweis erbracht werden, dass es sich um ausgereifte ORN handelte [67].

Da die Rate an neu entstandenen ORN bei Tsujigiwa et al. eher gering war, wollte eine weitere Arbeitsgruppe dies optimieren, indem sie ausschließlich Knochenmarkstammzellen transplantierten. Die Übertragung der mittels Bromdesoxyuridin (BrdU) markierten Stammzellen erfolgte bei einer Gruppe von Mäusen intravenös sowie bei einer anderen lokal mittels transnasaler Injektion. Immunhistochemisch konnten bei beiden Applikationsformen Zellen im Riechepithel gefunden werden, die positiv für GAP43 waren, was typischerweise von globosen Basalzellen exprimiert wird. Drei Wochen nach Transplantation waren die Zellen in der mittleren Schicht der Riechschleimhaut angelangt. Eine Ausdifferenzierung der Vorläuferzellen in reife ORN trat allerdings nicht ein. Das Ergebnis dieser Studie deutet außerdem an, dass die Effizienz der Zelltransplantationsanwachsrate bei lokaler und systemischer Gabe vergleichbar ist [49].

Der Effekt von Stammzellen wurde auch im Bereich des BO untersucht. Eine spanische Forschungsgruppe führte Therapieversuche mit Knochenmarkstammzellen bei transgenen Mäusen durch, die einen Verlust von Mitralzellen im Bulbus zeigen, also des zweiten Neurons der Riechbahn. Ein Verlust von Mitralzellen im Bulbus führt zu einer verminderten Geruchsdetektion und Geruchsdiskrimination der Mäuse. Die intravenöse Gabe von Knochenmarkzellen führte im BO zur Ausbildung von Mikrogliazellen, jedoch nicht von Mitralzellen oder anderen neuronalen Zellen. Auffallend war, dass bei den transplantierten Mäusen der normale progressive Verlust von Mitralzellen deutlich reduziert werden konnte. Folglich lässt sich annehmen, dass Mikrogliazellen eine protektive Wirkung in Bezug auf Mitralzellen zu haben scheinen. Aus früheren Arbeiten ist bereits bekannt, dass Mikrogliazellen neuroprotektive Substanzen freisetzen können. Mittels elektrophysiologischer Untersuchungen wurde außerdem deutlich, dass die transplantierten Mäuse im Ver- 
gleich zur Kontrollgruppe eine Verbesserung in der Geruchserkennung und der Geruchsdiskrimination zeigten [21].

Einen neuen Therapieansatz von Riechstörungen verfolgten Kurtenbach et al. durch die Transplantation von gewebespezifischen Stammzellen. Für ihre Untersuchung erstellten sie ein transgenes Mausmodel, bei welchem sie das interflagelläre Transportprotein aus der DNA der Mäuse entfernten, um bei den Tieren eine Hyposmie zu induzieren. Die Hyposmie wurde objektiviert, indem bei den behandelten Mäusen mittels Elektroolfaktogramm deutlich reduzierte elektrophysiologische Antworten auf Bananenduft (Amylacetat) zu messen waren. Als histologisches Korrelat fanden sie eine Ziliopathie der ORN. Hinsichtlich der transplantierten Stammzellen wurden ausschließlich Stammzellen der Riechschleimhaut verwendet, welche c-KIT-Oberflächenrezeptor-positiv waren. C-KIT-positive globose Basalzellen (GBC) haben die Fähigkeit zur Neurogenese. Um möglichst viele dieser Stammzellen für die Transplantation generieren zu können, wurden Spendermäuse zwei Tage vor Entnahme der Zellen mit Methimazol behandelt, was zu einem Zelltod von reifen ORN und gleichzeitig zu einer Hochregulierung von GBC führt. Die c-KIT-positiven GBC wurden mittels nasaler Injektion in die hyposmischen Mäuse eingebracht. Vier Wochen nach erfolgter Transplantation wurde die olfaktorische Schleimhaut histologisch untersucht: Aus den transplantierten Stammzellen bildeten sich zilientragende ORN. Ebenso konnte ein Axonwachstum im N. olfactorius nachgewiesen werden. Die Axonaussprossung reichte sogar bis in die Glomeruli des Bulbus. Periglomerulär fanden sich zudem tyrosinhydroxylasepositive Zellen, was auf eine duftinduzierte Zellaktivität hinweist. Im Weiteren zeigten sich in elektroolfaktographischen Messungen und auch in Verhaltenstests bessere Resultate der zellbehandelten Mäuse im Vergleich zur Kontrollgruppe. Die transplantierten Zellen scheinen also nicht nur in die Riechschleimhaut einzuwachsen und sich zu differenzieren, sondern auch zu einer Verbesserung der Riechfunktion zu führen [36].
Das Potenzial von Stammzelltherapien beim Einsatz von Riechstörungen scheint vielversprechend zu sein. Eine der großen Herausforderungen ist sicherlich der Sicherheitsaspekt bei solchen Therapieansätzen, insbesondere hinsichtlich unkontrollierter Zellentwicklung und Zellmigration mit entsprechendem Entartungsrisiko.

\section{Transplantation von Riechschleimhaut}

Neben der Zelltherapie gibt es auch Bestrebungen zur Verpflanzung von Riechschleimhaut im Zellverband. Bereits 1983 wurde olfaktorische Schleimhaut von neonatalen Ratten in den parietalen Kortex und in den vierten Ventrikel von adulten und neugeborenen Tieren transplantiert. Die Neurogenese blieb trotz der ektopen Lage der Schleimhaut vorhanden. Es ließen sich sogar Axonbündel nachweisen, die das Empfängerhirn penetrierten. $\mathrm{Zu}$ einer Ausbildung von olfaktorischen Glomeruli kam es jedoch nicht [43]. Holbrook et al. erreichten ähnliche Resultate mit Transplantaten von postnatalen Mäusen. Das Transplantat überlebte in $85 \%$ der Fälle [29].

Riechschleimhaut kann allerdings nicht nur in den Kortex, sondern auch in den BO transplantiert werden. Die Überlebensrate des Implantats war an beiden Lokalisationen gleich und betrug $83 \%$. Charakteristisch für die transplantierte olfaktorische Mukosa war das Auftreten von aus Epithelzellen bestehenden Vesikeln. In histologischen Untersuchungen wurde zudem ersichtlich, dass der mehrschichtige Aufbau der Riechschleimhaut erhalten blieb, ebenso konnten zilientragende Zellen an der Epitheloberfläche dargestellt werden, funktionelle Synapsen mit dem Bulbus jedoch nicht [79].

Insgesamt konnte also gezeigt werden, dass olfaktorische Schleimhaut in diverse Stellen des Kortex sowie in den vierten Ventrikel und den BO (zusammenfassend, gemeinsam mit den Ergebnissen der Stammzelltransplantationen, in - Tab. 2 dargestellt) transplantiert werden kann. Wie es um ihre Funktionalität in Bezug auf das Riechvermögen steht, wurde bisher in keiner Studie näher untersucht.

\section{Fazit und Ausblick}

Zusammenfassend zeigen sich einerseits klare Hinweise zur Machbarkeit der Entwicklung einer bioelektrischen Nase. Die direkte elektrische Stimulation des BO stellt möglicherweise eine Therapieoption zur Behandlung der Anosmie dar, wenn z. B. aufgrund irreversibler Schädigungen der ORN olfaktorische Informationen nicht an den $\mathrm{BO}$ weitergeleitet werden können, um schließlich in höher gelegenen Zentren verarbeitet zu werden. Unklar ist bislang jedoch, bis zu welchem Zeitpunkt, d.h. bis zu welcher Dauer der Riechstörung, eine elektrische Stimulation erfolgversprechend ist. Wahrscheinlich ist der Mensch in der Lage, Myriaden von Gerüchen zu unterscheiden [11]. Dies zeigt, dass die Ansprüche an ein etwaiges olfaktorisches Implantat groß sind. Es muss in der Lage sein, die verschiedenen olfaktorischen Informationen zu detektieren und duftspezifische, elektrische Signale an den BO weiterleiten. Hier ist allerdings bekannt, dass das Volumen des BO vom Alter und der olfaktorischen Funktion abhängig ist [10, $26,46,59]$. Es ist unklar, inwiefern dieser Aspekt Einfluss auf die Entwicklung eines olfaktorischen Implantats hat. Für die erfolgreiche Hör- und Sehrehabilitation mithilfe eines Cochlea- oder eines Retinaimplantats ist ein intakter Hör- bzw. Sehnerv eine Grundvoraussetzung. Die meisten Untersuchungen beziehen sich zwar ebenfalls auf die indirekte oder direkte elektrische Stimulation des N. olfactorius bzw. des BO, jedoch konnten Geruchsempfindungen auch durch eine subdurale [35], kortikale [40] und thalamische [48] Reizung generiert werden. Die bis dato durchgeführten elektrischen Stimulationen beim Menschen bedingten zum Teil operative Eingriffe bis hin zur Kraniotomie, was entsprechend invasiv und mit Risiken verbunden ist. Hinsichtlich der Invasivität wäre der Einsatz von Stammzellen klar zu präferieren, welche relativ einfach mittels nasaler Injektion eingebracht werden können. Der Einsatz von Stammzellen bei Riechstörungen geht bis jetzt aber noch nicht über Tier- 
modelle hinaus, obschon die Studienergebnisse, insbesondere die von Kurtenbach et al., vielversprechende Ergebnisse zeigten in Bezug auf eine mögliche funktionelle Wiederherstellung des Riechepithels. Beim Einsatz von Stammzellen ist aber das kanzerogene Potenzial eines solchen Therapieansatzes bedenkenswert.

\section{Korrespondenzadresse}

\section{Dr. med. P. Dörig}

Universitäts-HNO Klinik Basel

Petersgraben 4, 4031 Basel, Schweiz

patrick.doerig@usb.ch

\section{Einhaltung ethischer Richtlinien}

Interessenkonflikt. P. Dörig, N. Gunder, M. Witt, A. Welge-Lüssen und T. Hummel geben an, dass kein Interessenkonflikt besteht.

Für diesen Beitrag wurden von den Autoren keine Studien an Menschen oder Tieren durchgeführt. Für die aufgeführten Studien gelten die jeweils dort angegebenen ethischen Richtlinien.

\section{Literatur}

1. Andy OJ (1967) The amygdala and hippocampus in olfactory aura. Electroencephalogr Clin Neurophysiol 23:292

2. Ang GC (2005) History of skin transplantation. Clin Dermatol 23:320-324

3. Aronsohn E (1886) Experimentelle Untersuchungen zur Physiologie des Geruches. In: Archiv für Anatomie und Physiologie, S 321

4. Balassa K, Danby R, Rocha V (2019) Haematopoietic stem cell transplants: principles and indications. $\mathrm{Br}$ JHosp Med (Lond) 80:33-39

5. Berard N, Landis BN, Legrand Letal (2020) Electrical stimulation of the medial orbitofrontal cortex in humans elicits pleasant olfactory perceptions. Epilepsy Behav 114:107559

6. Besser G, Liu DT, Renner B et al (2019) Olfactory implant: demand for a future treatment option in patients with olfactory dysfunction. Laryngoscope 129:312-316

7. Bocksberger S, Wagner W, Hummel T et al (2020) Temporary hyposmia in COVID-19 patients. HNO 68:440-443

8. Bramerson A, Johansson L, Ek L et al (2004) Prevalence of olfactory dysfunction: the skovde population-based study. Laryngoscope 114:733-737

9. Buck LB (2000) Smell and taste: the chemical senses. In: Kandel ER, Schwartz JH, Jessell TM (Hrsg) Principles of neural science. McGraw-Hill, New York, S625-652

10. Buschhuter D, Smitka M, Puschmann S et al (2008) Correlation between olfactory bulb volume and olfactory function. Neuroimage 42:498-502

11. Bushdid C, Magnasco MO, Vosshall LB et al (2014) Humans can discriminate more than 1 trillion olfactory stimuli. Science 343:1370-1372

12. Butowt R, Von Bartheld CS (2020) Anosmia in COVID-19: underlying mechanisms and as- sessment of an olfactory route to brain infection. Neuroscientist. https://doi.org/10.1177/ 1073858420956905

13. Child KM, Herrick DB, Schwob JE et al (2018) The neuroregenerative capacity of olfactory stem cells is not limitless: implications for aging. J Neurosci 38:6806-6824

14. Coelho DH, Costanzo RM (2016) Spatial mapping in the rat olfactory bulb by odor and direct electrical stimulation. Otolaryngol Head Neck Surg 155:526-532

15. Coleman JH, Lin B, Louie JD et al (2019) Spatial determination of neuronal diversification in the olfactory epithelium. J Neurosci 39:814-832

16. Costanzo RM, Yagi S (2011) Olfactory epithelial transplantation: possible mechanism for restoration of smell. Curr Opin Otolaryngol Head Neck Surg 19:54-57

17. Croy I, Nordin S, Hummel T (2014) Olfactory disorders and quality of life - an updated review. Chem Senses 39:185-194

18. Dai Q, Pang Z, Yu H (2016) Recovery of olfactory function in postviral olfactory dysfunction patients after acupuncture treatment. Evid Based Complement Alternat Med 2016:4986034

19. Damm M, Pikart LK, Reimann H et al (2014) Olfactory training is helpful in postinfectious olfactory loss: a randomized, controlled, multicenter study. Laryngoscope 124:826-831

20. Damm M, Schmitl L, Muller CA et al (2019) Diagnostics and treatment of olfactory dysfunction. HNO 67:274-281

21. Diaz D, Lepousez G, Gheusi G et al (2012) Bone marrow cell transplantation restores olfaction in the degenerated olfactory bulb. J Neurosci 32:9053-9058

22. Dong Q, Du L, Zhuang L et al (2013) A novel bioelectronic nose based on brain-machine interface using implanted electrode recording in vivo in olfactory bulb. Biosens Bioelectron 49:263-269

23. Durante MA, Kurtenbach S, Sargi ZB et al (2020) Single-cell analysis of olfactory neurogenesis and differentiation in adult humans. Nat Neurosci 23:323-326

24. Gilad Y, Man O, Paabo Set al (2003) Human specific loss of olfactory receptor genes. Proc Natl Acad Sci USA 100:3324-3327

25. Graziadei PP, Monti Graziadei AG (1983) Regeneration in the olfactory system of vertebrates. Am J Otolaryngol 4:228-233

26. Gudziol V, Buschhuter D, Abolmaali N et al (2009) Increasing olfactory bulb volume due to treatment of chronic rhinosinusitis - a longitudinal study. Brain 132:3096-3101

27. Hatzinger M, Stastny M, Grutzmacher Pet al (2016) The history of kidney transplantation. Urologe $A$ 55:1353-1359

28. Henkin RI, Potolicchio SJ Jr., Levy LM (2011) Improvement in smell and taste dysfunction after repetitive transcranial magnetic stimulation. Am J Otolaryngol 32:38-46

29. Holbrook EH, Dinardo LJ, Costanzo RM (2001) Olfactory epithelium grafts in the cerebral cortex: an immunohistochemical analysis. Laryngoscope 111:1964-1969

30. Holbrook EH, Puram SV, See RB et al (2019) Induction of smell through transethmoid electrical stimulation of the olfactory bulb. Int Forum Allergy Rhinol 9:158-164

31. Hummel T, Heilmann S, Huttenbriuk KB (2002) Lipoic acid in the treatment of smell dysfunction following viral infection of the upper respiratory tract. Laryngoscope 112:2076-2080
32. Isenmann S, Haehner A, Hummel T (2021) Chemosensory disorders in Covid-19: pathomechanisms and clinical relevance. Fortschr Neurol Psychiatr. https://doi.org/10.1055/a-1375-0761

33. Ishimaru T, Shimada T, Sakumoto M et al (1997) Olfactory evoked potential produced by electrical stimulation of the human olfactory mucosa. Chem Senses 22:77-81

34. Kadohisa M (2013) Effects of odor on emotion, with implications. Front Syst Neurosci 7:66

35. Kumar G, Juhasz C, Sood S et al (2012) Olfactory hallucinations elicited by electrical stimulation via subdural electrodes: effects of direct stimulation of olfactory bulb and tract. Epilepsy Behav 24:264-268

36. Kurtenbach S, Goss GM, Goncalves S et al (2019) Cell-based therapy restores olfactory function in an inducible model of hyposmia. Stem Cell Reports 12:1354-1365

37. Landis BN, Konnerth CG, Hummel T (2004) A study on the frequency of olfactory dysfunction. Laryngoscope 114:1764-1769

38. Leopold DA, Loehrl TA, Schwob JE (2002) Longterm follow-up of surgically treated phantosmia. Arch Otolaryngol Head Neck Surg 128:642-647

39. Malnic B, Hirono J, Sato Tet al (1999) Combinatorial receptor codes for odors. Cell 96:713-723

40. Mazzola L, Royet JP, Catenoix H et al (2017) Gustatory and olfactory responses to stimulation of the human insula. Ann Neurol 82:360-370

41. Meisami $E$ (1976) Effects of olfactory deprivation on postnatal growth of the rat olfactory bulb utilizing a new method for production of neonatal unilateral anosmia. Brain Res 107:437-444

42. Mombaerts P (1999) Seven-transmembrane proteins as odorant and chemosensory receptors. Science 286:707-711

43. Morrison EE, Graziadei PP (1983) Transplants of olfactory mucosa in the rat brain I. A light microscopic study of transplant organization. Brain Res 279:241-245

44. Morrissey DK, Pratap U, Brown C et al (2016) The role of surgery in the management of phantosmia. Laryngoscope 126:575-578

45. Mudry A, Mills M (2013) The early history of the cochlear implant: a retrospective. JAMA Otolaryngol Head Neck Surg 139:446-453

46. Mueller A, Rodewald A, Reden J et al (2005) Reduced olfactory bulb volume in post-traumatic and post-infectious olfactory dysfunction. NeuroReport 16:475-478

47. Murrell W, Bushell GR, Livesey J et al (1996) Neurogenesis in adult human. NeuroReport 7:1189-1194

48. Nashold BS, Wilson WP (1970) Olfactory hallucinations evoked from stimulation of human thalamus. Confin Neurol 32:298-306

49. Ochi N, Doi K, Uranagase M et al (2010) Bone marrow stem cell transplantation to olfactory epithelium. Ann Otol Rhinol Laryngol 119:535-540

50. Otte MS, Klussmann JP, Luers JC (2020) Smell disorders at COVID-19-the current level of knowledge. Laryngorhinootologie 99:531-535

51. Ottoson D (1959) Olfactory bulb potentials induced by electrical stimulation of the nasal mucosa in the frog. Acta Physiol Scand 47:160-172

52. Penfield W, Jasper H (1954) Epilepsy and the functional anatomy of the human brain. Little Brown, Boston

53. Peterson NR, Pisoni DB, Miyamoto RT (2010) Cochlear implants and spoken language processing abilities: review and assessment of the literature. Restor Neurol Neurosci 28:237-250 
54. Philpott CM, Boak D (2014) The impact of olfactory disorders in the United Kingdom. Chem Senses 39:711-718

55. Qiao XF, Wang GP, Li X et al (2019) Analysis of the clinical effect of olfactory training on olfactory dysfunction after upper respiratory tract infection. Acta Otolaryngol 139:643-646

56. Quint C, Temmel AF, Hummel T et al (2002) The quinoxaline derivative caroverine in the treatment of sensorineural smell disorders: a proof-ofconcept study. Acta Otolaryngol 122:877-881

57. Rachitskaya AV, Yuan A (2016) Argus II retinal prosthesis system: an update. Ophthalmic Genet 37:260-266

58. Ressler KJ, Sullivan SL, Buck LB (1994) Information coding in the olfactory system: evidence for a stereotyped and highly organized epitope map in the olfactory bulb. Cell 79:1245-1255

59. Rombaux P, Potier H, Markessis E et al (2010) Olfactory bulb volume and depth of olfactory sulcus in patients with idiopathic olfactory loss. Eur Arch Otorhinolaryngol 267:1551-1556

60. Saussez S, Lechien JR, Hopkins C (2020) Anosmia: an evolution of our understanding of its importance in COVID-19 and what questions remain to be answered. Eur Arch Otorhinolaryngol. https://doi. org/10.1007/s00405-020-06285-0

61. Schriever VA, Merkonidis C, Gupta N et al (2012) Treatment of smell loss with systemic methylprednisolone. Rhinology 50:284-289

62. Schwob JE (2002) Neural regeneration and the peripheral olfactory system. Anat Rec 269:33-49

63. Serizawa S, Ishii T, Nakatani H et al (2000) Mutually exclusive expression of odorant receptor transgenes. Nat Neurosci 3:687-693

64. Sharma A, KumarR, Aierletal (2019) Sense of smell: structural, functional, mechanistic advancements and challenges in human olfactory research. Curr Neuropharmacol 17:891-911

65. Sorokowska A, Drechsler E, Karwowski M et al (2017) Effects of olfactory training: a meta-analysis. Rhinology 55:17-26

66. Straschill M, Stahl H, Gorkisch K (1983) Effects of electrical stimulation of the human olfactory mucosa. Appl Neurophysiol 46:286-289

67. Tsujigiwa H, Nishizaki K, Teshima T et al (2005) The engraftment of transplanted bone marrowderived cells into the olfactory epithelium. Brain Res 1052:10-15

68. Uchida N, Takahashi YK, Tanifuji Metal (2000) Odo maps in the mammalian olfactory bulb: domain organization and odorant structural features. Nat Neurosci 3:1035-1043

69. Uziel A (1973) Stimulation of human olfactory neuro-epithelium by long-term continuous electrical currents. J Physiol (Paris) 66:409-422

70. Van Der Linden CJ, Gupta P, Bhuiya Al et al (2020) Olfactory stimulation regulates the birth of neurons that express specific odorant receptors. Cell Rep 33:108210

71. Vassar R, Chao SK, Sitcheran R et al (1994) Topographic organization of sensory projections to the olfactory bulb. Cell 79:981-991

72. Vassar R, Ngai J, Axel R (1993) Spatial segregation of odorant receptor expression in the mammalian olfactory epithelium. Cell 74:309-318

73. Vennemann MM, Hummel T, Berger K (2008) The association between smoking and smell and taste impairment in the general population. J Neurol 255:1121-1126

74. Verbeurgt C, Wilkin F, Tarabichi M et al (2014) Profiling of olfactory receptor gene expression in whole human olfactory mucosa. PLOS ONE 9:e96333
75. Weiss T, Shushan S, Ravia A et al (2016) From nose to brain: un-sensed electrical currents applied in the nose alter activity in deep brain structures. Cereb Cortex 26:4180-4191

76. Whitcroft KL, Cuevas M, Haehner A et al (2017) Patterns of olfactoryimpairment reflect underlying disease etiology. Laryngoscope 127:291-295

77. Witt M (2020) Anatomy and development of the human gustatory and olfactory system. In: Fritzsch B (Hrsg) The senses: a comprehensive reference, 2. Aufl. Elsevier, Amsterdam, S85-118

78. Witt M, Hansen A (2009) Strukturelle und funktionelle Grundlagen des Riechens. In: Hummel T, Welge-Luessen A (Hrsg) Riech- und Schmeckstörungen. Thieme, Stuttgart, S11-26

79. Yagi S, Costanzo RM (2009) Grafting the olfactory epithelium to the olfactory bulb. Am J Rhinol Allergy 23:239-243

Aktuelle Informationen

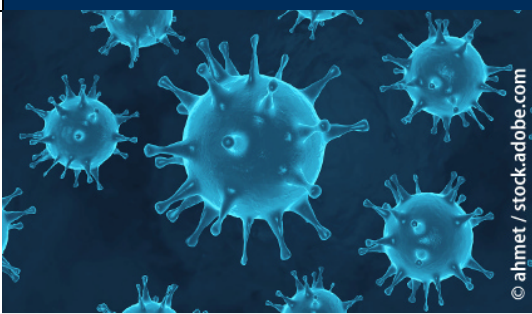

Aktuelle Entwicklung zu COVID-19 bei Springer Nature und Springer Medizin

Springer Nature und Springer Medizin unterstützen die globale Reaktion auf die COVID-19-Pandemie, indem ein schneller und direkter $\mathrm{Zu}$ gang zu den neuesten verfügbaren Forschungsergebnissen und Daten ermöglicht wird.

Auf der Homepage SpringerMedizin.de finden Sie ein immer aktuelles Dossier mit Beiträgen, Forschungsarbeiten und Ergebnissen zu SARS-CoV-2 sowie relevanten Links.

Darin z.B. auch die publizierte Empfehlung von DIVI, DGIIN, DGAI und DGP zur Intensivtherapie von Patienten mit COVID-19.

Springer Nature arbeitet mit globalen Organisationen zusammen, und verlinkt über SpringerNature.com/de auf eine eigene Landingpage mit einer Vielzahl an Information sowie freiem Zugriff auf die COVID-19-Contentplattformen von Nature Research, BioMed Central (BMC) und Springer.

Das Dossier zu Coronavirus / Covid-19 von Springer Medizin finden Sie hier: www.springermedizin.de/covid-19

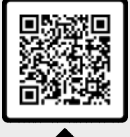

